\title{
Recycle spent batteries
}

\author{
The rapid growth of lithium-ion batteries in many markets makes it increasingly urgent to address recycling of \\ strategic materials from spent batteries.
}

B atteries typically contain toxic chemicals and cannot be disposed of at will. The European Battery Directive states that all batteries must be collected and recycled; in practice it is up to European Union member states to determine implementation. The United Kingdom, for example, has reportedly struggled to meet battery recycling targets ${ }^{1}$. Most batteries collected for recycling are lead-acid batteries, an old battery technology that is being replaced by lithium-ion batteries (LIBs). LIBs are currently recycled at a meagre rate of less than 5\% in the European Union $^{2}$. This is alarmingly low considering the recent staggering growth of LIBs in electrified transport, which has seen a nearly 20 -fold increase in electric vehicle (EV) sales in just 5 years. Given that EV manufacturers typically offer 8 year/100,000 mile warranties for their batteries, the rapid increase in EVs will translate into ever more waste batteries. Meanwhile, the development of electric aircraft on the horizon ${ }^{3}$ and their huge battery demands will put even more pressure on future battery recycling.

Most EVs are powered by LIBs because of their relatively high energy density as well as the diverse range of power-to-energy ratios they offer. There are also many different LIBs depending on the materials used for cathodes, anodes, electrolytes, separators and other accessories. Take cathodes, a major costly component and a bottleneck for energy density, as an example: lithium nickel cobalt aluminium oxides are preferred by Tesla, whereas lithium nickel manganese oxides with a range of compositions have been adopted by many other EV makers; lithium iron phosphate, a less energy-dense but inexpensive cathode, is used by EV makers in regions such as China; blending different cathode materials is also practiced in manufacturing.

The versatility of battery components and their rich chemistry thus pose serious challenges for recycling. In industry, the most common LIB recycling techniques use extractive metallurgical processes, including pyrometallurgy and hydrometallurgy, to recycle valuable but scarce metals from cathode materials. However, pyrometallurgy needs high-temperatures and has a low recovery rate, while hydrometallurgy consumes lots of chemical reagents and requires a long recovery process. More environmentally friendly mechanical approaches exist, but their efficacy is far from perfect.

Laboratory research can offer new ideas and alternatives. In their Article in this issue, Pulickel Ajayan and team report a method of using deep eutectic solvents to dissolve and extract valuable metals from cathode materials including lithium cobalt oxides and lithium nickel manganese oxides, with cobalt and lithium recovery rates of up to $90 \%$. The solvents use biodegradable precursors that can be reused over multiple cycles.

For a recycling technique to be viable it must have economic or environmental value. In this respect, the emerging concept of direct recycling could be beneficial ${ }^{4}$. Direct recycling recovers cathode materials as reusable cathode mixtures instead of individual metals, in principle minimizing waste produced during recycling. However, direct recycling targets specific cathodes and thus requires specialized processes for different cathodes. Furthermore, its recovery efficiency depends on the battery state of health and so may not be advantageous if the state-of-charge is too low.

Alongside direct recycling, seconduse applications can prolong the life of spent EV batteries. Repurposed batteries can be adopted for the electricity grid for applications such as peak shaving or in combination with renewable energy installation in buildings to increase energy self-consumption. While some pilot and research projects on second-use applications are showing environmental benefits, uncertainties remain around the economic aspects due to a lack of data availability and quality ${ }^{5}$.

The economic uncertainty has also much to do with the supply chain of battery raw materials, which is heavily influenced by their market prices. Recent years have seen large cobalt and lithium price fluctuations both, arguably the most important strategic LIB materials, raising questions as to whether battery recycling or second-use applications are economically beneficial compared with using fresh batteries. The economics get murkier still if we also consider the impact of next-generation technologies such as solid-state and sodiumion batteries, which could shift the focus to different strategic materials.
While most current recycling efforts focus on cathode materials due to the expected economic incentives towards valuable metals, little has been reported on the recovery of anodes, electrolytes and other LIB components. Yet anodes could be as expensive as cathodes, especially in lithium metal batteries whose anodes require a lot more lithium than in LIBs; while most electrolytes in current LIBs contain hazardous organic materials and need to be recycled, solid electrolytes in solid-state batteries will become a new strategic material. As lithium metal and solid-state batteries are widely tipped for near-future EV applications, new recycling challenges should be anticipated so that new recycling technologies are in place when needed.

The LIB recycling and repurposing industry is currently very small compared with the vast and rapidly increasing battery manufacturing industry, yet encouraging developments are beginning to emerge. This year has seen the launch of the first battery recycling research centre by the US Department of Energy, which has the recycling goal of profitably capturing $90 \%$ of lithium-based battery technologies ${ }^{6}$. Meanwhile, EV makers are partnering with energy storage firms to create new business models for second-life applications ${ }^{7}$. Concerted efforts like these, will help address the challenges in recycling technologies and uncertainty concerning added values of repurposing applications.

Published online: 11 April 2019

https://doi.org/10.1038/s41560-019-0376-4

References

1. Doherty, J. Concern as UK continues to struggle on battery recycling target. letsrecycle.com https://go.nature.com/2OpIofS (2018).

2. Gardiner, J. The rise of electric cars could leave us with a big battery waste problem. The Guardian (10 August 2017); https://go.nature.com/2JG9NeO

3. Schäfer, A. W. et al. Nat. Energy 4, 160-166 (2019).

4. Ciez, R. E. \& Whitacre, J. F. Nat. Sustain. 2, 148-156 (2019).

5. Bobba, S. et al. Sustainability Assessment of Second Life Application of Automotive Batteries (SASLAB): JRC Exploratory Research (2016-2017): Final Technical Report, August 2018 (Publications Office of the European Union, 2018); https://go.nature.com/2Ovl68s

6. Energy Department announces battery recycling prize and battery recycling R\&D center. Energy.gov https://go.nature. $\mathrm{com} / 2 \mathrm{uCkc0v}$ (2019).

7. Field, K. Hyundai partners with Wärtsilä on stationary storage using second-life batteries. CleanTechnica https://go.nature. com/2Wo4RMX (2018) 